\title{
- Modern Therapeutics Applications
}

\section{Suryakant Nagtilak}

Professor and HOD, Department of Biochemistry, Govt. Medical College Silvassa, Dadra and Nagar Haveli, India.

Email:nagtilak@yahoo.com

Sci. Journal Impact

Factor: 6.1 (2018)

ICV: $90.90(2018)$

Scopus'

Current modalities of diagnosis and treatment of various diseases have major limitations such as selectivity and specificity. Over the eons, the worldwide health care system has experienced unprecedented change and growth. These involved dramatic innovations in the technology of medicine, evolving expectations on the part of the consumer (patients and their family) toward physicians. Physician involving general practitioners to surgeons, nurses to physiotherapist, dramatically adapted to these changes as and when needed. These health care practitioners contributed significantly for betterment of the system that ultimately resulted into availability of various options for the patients for the speedy recovery. The present special issue, 'Novel medical applications' is based on the nature of these amendments, their anticipated future course, and the implications for the physician, the consumer (patients and their family), and the overall health care system.

Modern therapeutic application is changing the way of thinking of population related to family planning to giving prenatal stimulation to baby in the womb via mother to awareness about the diseases and its correlation with individual's routine. Implication of different scoring system to diagnose the disease and its correlation with the therapeutic outcomes. Replacing the traditional diagnostic method by novel one for the disease may also be beneficial for the precise diagnosis. A research article compared the usefulness of MRI and Arthroscopy examination in detecting ACL injuries. A try for the use of rigid brochoscope for the therapeutic bronchoscopy was tried on 13 patients and its merits in the management of trachea-bronchial foreign bodies is suggested. An article is also focused on the importance of self-care in terms of pathogens present on the widely used gadgets such as cell phone. Furthermore, MRSA infection is commonly seen in healthcare personnel and essential prevention strategies are reviewed. Health care fraternity must be aware of this to avoid the possible infection due to these pathogens. Most of the surgeons use the advanced techniques to get the maximum effect of traditional approaches. One such approach is distal end clavicle fractures treated with locking plates.
The use of clonidine as adjuvant to infiltration anaesthesia in tympanoplasty surgery, nalbuphine as an addition to anaesthesia armamentarium to prolong postoperative analgesia and the use of intracameral adrenaline bolus injection to control the pupil size, pulse rate and blood pressure during small incision cataract surgery are the novel approach to facilitate the common clinical surgeries. This also indicated the novel use of commonly practiced drug in surgical hospitals. Many advancements for clinical investigations for the CNS related disorder is also happening. Clinical spectrum of obsessivecompulsive disorder in different cohort of population is studied. One considering the patients from central India is also published in this special issue which report in their study the higher prevalence of disease in the young individuals from lower socioeconomic class.

Review on effect of obesity and related complications, role of minerals like zinc and vitamin in overall health is vital and this is very well disseminated by physicians to the different cohorts of population. Broader sense of commonly known and high prevalent disease such as diabetes is also presented. This is compared with Prameha and ayurvedic approach to treat the both is suggested in the article present here. It is common practice to titrate the drug and dose as per the physiological status and diagnosis of the disease. This is called as precision medicine. One such article suggested that diclofenac is more effective than tramadol in the treatment of ureteric colic patients. Novel indication of old drugs is suggested wherein use of prostaglandin E2 is proposed for the cervical ripening in labour induction.

I conclude that modern therapeutic applications are wisely implemented but health care personals in the diagnosis and clinical setup and that ultimately improve the recovery form the disease as well as provide the quality of life despite illness to patients and their families. This also reduced the social burden of the disease and all credit goes to physicians and other member of health care system. This special issue is about the highlights of some of these advancement by the personals of health care system. 